\title{
Diversidad de anuros en humedales del Centro de Investigación Amazónica Macagual, Florencia, Caquetá, Colombia
}

\section{Diversity of anuros in wetlands of the Center of Amazonian Investigation Macagual, Florencia, Caquetá, Colombia}

\author{
Nayra Camila Arriaga-Villegas ${ }^{1}$, Natalia Andrea Obregon-Paz ${ }^{1}$, \\ Diego Huseth Ruiz-Valderrama ${ }^{2}$
}

\begin{abstract}
Resumen
Objetivo: Se determinó la riqueza y la abundancia para realizar una comparación de los anuros en dos humedales del Centro de Investigación Macagual (Caquetá, Colombia). Metodología: Se utilizó la técnica de captura que consistió en la búsqueda intensiva tanto auditiva como visual, entre marzo y noviembre del año 2012. Resultados: Se registraron un total de 144 individuos, distribuidos en seis especies, cuatro géneros y dos familias (Hylidae y Leptodactylidae). La mayor dominancia, según el índice de Simpson (D), la obtuvo el humedal de potrero. La mayor equitatividad (1-D) se encontró en el humedal de borde de bosque y mediante el índice de Margalef se obtuvo que la mayor riqueza la presentó el humedal de borde de bosque, debido a la heterogeneidad vegetal, generando condiciones climáticas óptimas para los anuros, determinando nichos más restringidos y más especies por unidad de área. Estas condiciones favorecen la diversidad y abundancia de anuros porque les confieren diversos sitios de refugio, alimentación y oviposición. Conclusión: El humedal de potrero presentó mayor abundancia, con 75 individuos y mayor dominancia; en cuanto al humedal de borde de bosque, se capturaron 69 individuos y se obtuvo mayor diversidad, por una mejor distribución y homogeneidad del espacio.
\end{abstract}

Palabras clave: Abundancia, Anuros, Humedales, Riqueza.

\begin{abstract}
Objective: Richness, abundance was determined and a comparison of anurans was conducted in two Macagual Wetlands Research Center (Caquetá, Colombia). Methodology: the capture technique that consisted thorough intensive search both auditory and visual, for the year 2012, from March to November. Results: registering a total of 144 individuals, divided into six species, four genera and two families (Hylidae and Leptodactylidae). The greater dominance as the Simpson index (D), obtained Wetland Potrero. Most Evenness (1-D) was found in the Wetland Edge Forest and by Margalef index which shows wealth, obtained Wetland Forest, due to plant heterogeneity, creating optimal climatic conditions for anurans, determining narrower niches and more species per unit area. These conditions favor the diversity and abundance of frogs and various sites that give them shelter, food and oviposition. Conclusion: The present Potrero wetland greater abundance, with 75 individuals and greater dominance, in terms of Wetland Forest Edge, 69 individuals were captured and greater diversity was obtained due to a better distribution or homogeneity of space.
\end{abstract}

Keywords: Abundance, Anuros, Wealth, Wetlands.

\section{Introducción}

Los ecosistemas acuáticos son componentes fun- damentales dentro de las organizaciones biológicas donde hay una gran integración entre elementos bióticos y abióticos (Urbina-Cardona 2009). Entre

\footnotetext{
Estudiantes de IX semestre de Biología, Semillero de Investigación en Herpetología, Facultad de Ciencias Básicas, Universidad de la Amazonia, Florencia, Caquetá. e-mail: bionairacamila@gmail.com

2 Biólogo, Investigador Asociado Museo de Historia Natural UAM, Grupo de Investigación en Fauna Silvestre, Universidad de la Amazonia, Florencia, Caquetá. Fecha recepción: Marzo 13, 2014 
estos ecosistemas acuáticos están los humedales como los más productivos del planeta, donde cuyo componente fundamental es el agua, en torno a la cual se forman ambientes intermedios que varían entre permanentemente inundados y normalmente secos (Castellanos 2001).

Los humedales amazónicos son considerados ecosistemas con múltiples características adecuadas para el mantenimiento de la biodiversidad tropical (Moreno et al. 2007), además poseen una alta diversidad y abundancia de especies tanto de flora y fauna que presentan ciertas funciones importantes dentro de estos ecosistemas acuáticos. También es importante reconocer que son hábitats de gran fragilidad asociadas con causas naturales como el cambio climático, las sequías prolongadas y la intervención humana, como en los casos de agricultura, pastoreo excesivo o el desgaste permanente por el ganado sobre el suelo (Pangea 2007). Las características de estos ecosistemas favorecen la diversidad y abundancia de anuros, porque les confieren diversos sitios de refugio, alimentación y oviposición (Lanno y Donnelly 2005).

Los anuros presentan un desarrollo de forma directa e indirecta, una doble vida, acuática y terrestre, algunos pueden ser acuáticos durante toda la vida y otros totalmente terrestres; estos últimos solo dependen de ambientes con algún grado de humedad para su reproducción. Estas formas de vida favorecen el transporte de energía y nutrientes, desempeñando un rol «pivot» entre ambos ambientes (AlfordRoss y Stephen-Richards 1999). Durante la etapa adulta, los individuos se mueven entre sitios terrestres de refugio o forrajeo y sitios acuáticos para la reproducción mientras que los juveniles utilizan ambientes semiacuáticos para dispersarse (BeebeeTrevor 1996). Es por ello que se encuentran formando parte de los humedales como sitios de refugio y reproducción (Lanno y Donnelly 2005), además los anuros son un orden de gran importancia como indicador de la intervención de estos ecosistemas, enfrentando grandes declinaciones en sus poblaciones a nivel global y, a pesar de tener una distribución muy amplia, alcanzan a colonizar todo tipo de hábitats (Acosta-Galvis 2000). Son un componente importante de la fauna de zonas de inundación (humedales) contribuyendo al reciclaje rápido de los detritos y el lodo (Rueda-Almonacid 1999).

En cuanto a la distribución y abundancia de las especies de anuros y su composición dentro de los humedales, estarían limitadas por condiciones ambientales tales como los regímenes de humedad y temperatura, la disponibilidad de nutrientes y la estructura física del hábitat (Begon et al. 2005). La comprensión de los patrones y procesos que influyen la riqueza y composición de especies es un objetivo continuo de la investigación en ecología (Baber y Babbi 2003). Las diferencias en los patrones de actividad y ciclos reproductivos de las distintas entidades biológicas suelen jugar un rol importante en la estructura temporal de los ensambles herpetológicos, y estas diferencias resultan en distintos ensambles a lo largo del tiempo (Fitzgerald et al. 1999). Por tanto, bajo el desarrollo de esta investigación se determinó la diversidad y composición de anuros en dos humedales con características o condiciones distintas del Centro de Investigación Amazónica Macagual en Florencia, Caquetá.

\section{Metodología}

Área estudio. El estudio se realizó en el Centro de Investigaciones Amazónicas Macagual (CIMAZ) ubicada a $20 \mathrm{~km}$ de Florencia, al sur del departamento del Caquetá, localizado geográficamente en la Amazonia colombiana a $1^{\circ} 37^{\prime} \mathrm{N}$ y $75^{\circ} 36^{\prime} \mathrm{W}$, a una altura de $300 \mathrm{msnm}$ (Figura 1). Hace parte de un bosque húmedo tropical con una temperatura promedio de $25.5^{\circ} \mathrm{C}$, una humedad relativa promedia de $76.3 \%$ y una precipitación de $3.793 \mathrm{~mm}$ al año (Estrada-Gonzáles y Rosas-Patiño 2007). Actualmente el centro de investigaciones está compuesto por bosque fragmentado con vegetación secundaria inmersos en un mosaico de pastizales y cultivos.

En el CIMAZ se encuentran una gran variedad de humedales, cada uno de ellos característicos por su ubicación, la cobertura vegetal que los rodea, por estar cubiertos de aguas en forma permanente, de profundidades media y de extensiones variables, por ende, las áreas de estudio, fueron dos humedales de particularidades diferentes; el primero está ubicado cerca al bosque y el segundo en un potrero con árboles dispersos. 


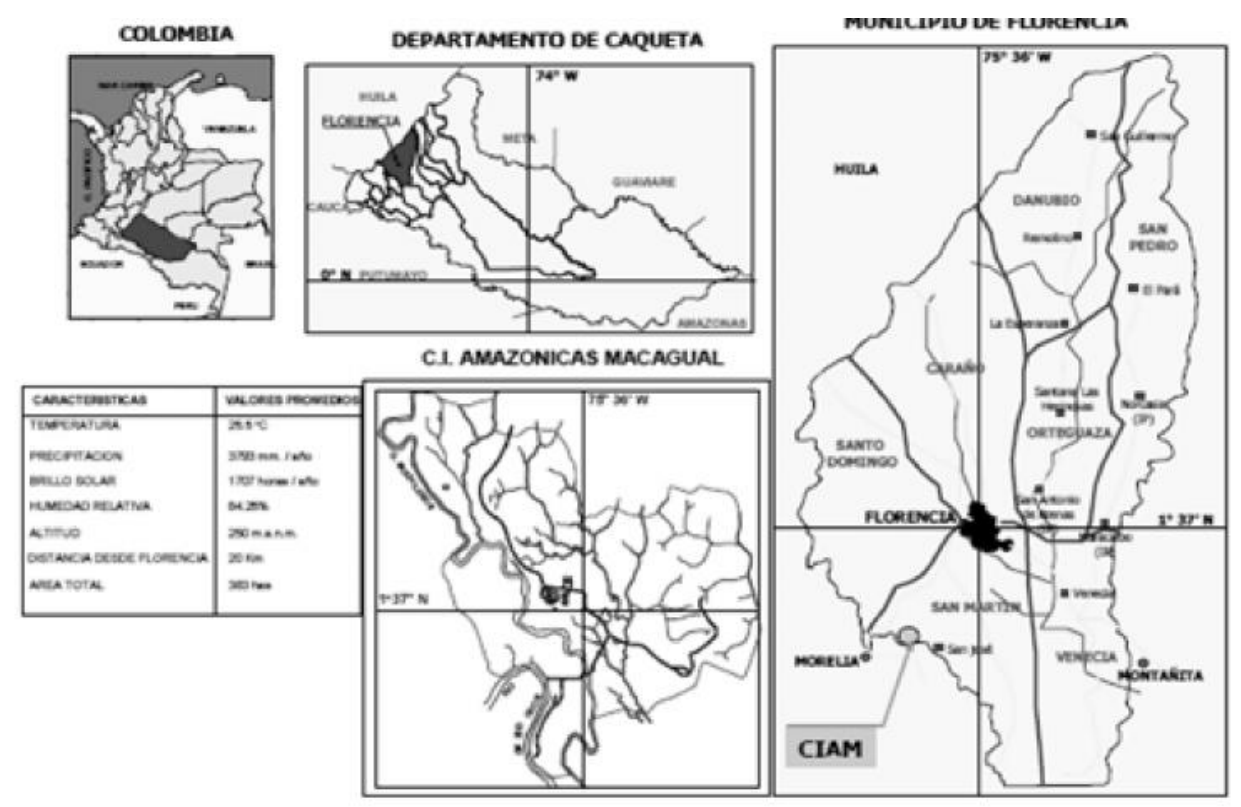

Figura 1. Ubicación del área de muestreo: Centro de Investigaciones Amazónica Macagual (CIMAZ), Caquetá, Colombia.

\section{Métodos}

Fase de campo. La investigación se realizó en el año 2012, desde el mes de marzo hasta noviembre (total de seis muestreos), registrándose las especies de anuros en estado juvenil y adulto observadas durante las horas de la noche para cada humedal, desde las 20:00 hasta las 23:00; teniéndose en cuenta estos horarios debido a las variaciones diarias del clima que pueden afectar la actividad de estas especies, como la temperatura y la radiación que son factores determinantes en los ritmos de actividad, al igual que la humedad donde juega quizá el peso más predominante; por tanto, se refleja que los picos de actividad de estos individuos están en la noche, aun más cuando se refiere a los anuros de humedales que tienen una alta fidelidad a estos sitios de reproducción y dispersión (Manzanilla y Pefaur 2000).

La técnica usada para la captura de anuros consistió en una búsqueda intensiva tanto auditiva como visual (Scott 1994); se capturaron manualmente, se introdujeron en bolsas de telas y después de acabada la jornada se identificaron luego de registrar la abundancia y toma de fotografías, se liberaron en el respectivo humedal del que fueron capturados.

Análisis de datos. La evaluación de la diversidad alfa se estableció mediante el programa estadís- tico PAST versión 2.15 (http://folk.uio.no/ohammer/ past. Oyvind Hammer, DAT Harper, abril 2012, Copyright Hammer and Harper 1999-2011), donde se analizó la dominancia; la abundancia se analizó con el índice de Simpson; la riqueza con el índice de Margalef; la diversidad con el índice ShannonWiener y la equitatividad con el índice de Simpson (1-D).

Se realizaron curvas de la distribución de la abundancia de los anuros presentes y tablas de distribución de la riqueza y abundancia de especies capturadas en cada uno de los humedales.

\section{Resultados}

Se registró un total de 144 individuos, distribuidos en seis especies, cuatro géneros y dos familias (Hylidae y Leptodactylidae). Hylidae fue la familia más representativa con cinco especies correspondiente a tres géneros (Dendropsophus, Scinax e Hypsiboas), la familia Leptodactylidae con un solo género (Leptodactylus) y una sola especie.

La dominancia de individuos para los dos humedales se concentró principalmente en dos especies Dendropsophus mathiassoni (62) y D. triangulum (33). Tres especies son intermedias Hypsiboas punctatus, Scinax garbey, H. cinerascens 
y solo una especie (Leptodactylus mystaceus) presentó una baja abundancia (Figura 2).

La dominancia de pocas especies y la alta proporción de especies con abundancias intermedias y raras, permiten que la curva de la distribución de la abundancia presente el modelo de serie logarítmica $\left(\chi^{2}=83,72 ; p=4,83\right.$ E-018; $\left.\alpha=0,05\right)$.

El humedal del potrero (HP) presentó la mayor abundancia, capturándose 75 individuos que conciernen a cinco especies en total, donde cuatro son de la familia Hylidae y una de Leptodactylidae. La especie más abundante fue $D$. mathiassoni $(65,3 \%)$ de anuros presentes en este humedal, seguido por $S$. garbey $(13,3 \%), H$. cinerascens $(10,6 \%), D$. triangulum $(9,3 \%)$ y L. mystaceus especie que presentó la menor abundancia (Figura 3).

En cuanto al humedal de borde de bosque (HBB) se capturaron 69 individuos pertenecientes a la familia Hylidae y del cual la especie más abundante fue $D$. triangulum, con $37,6 \%$ de la abundancia to-

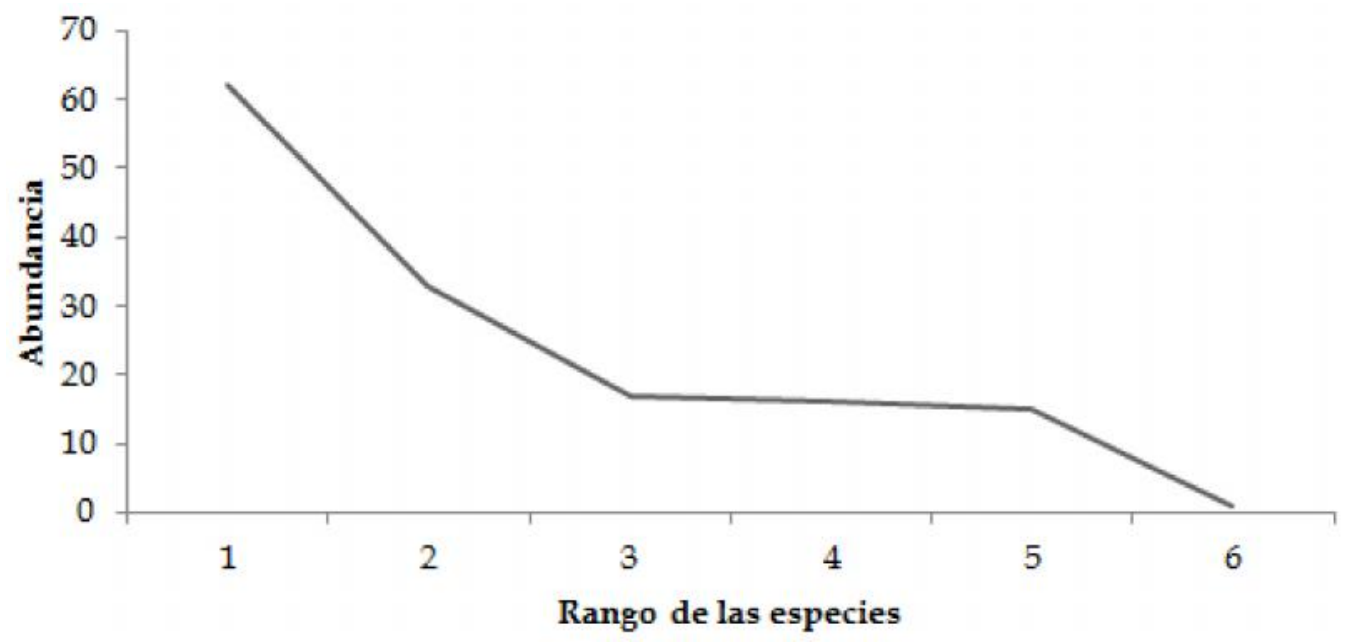

Figura 2. Curva de la distribución de la abundancia de los anuros presentes en los dos humedales del Centro de Investigación Amazónica Macagual, durante el año 2012. Modelo de distribución serie logarítmica $\left(\chi^{2}=83,72 ; p=4,83 E-018 ; \alpha=0,05\right)$.

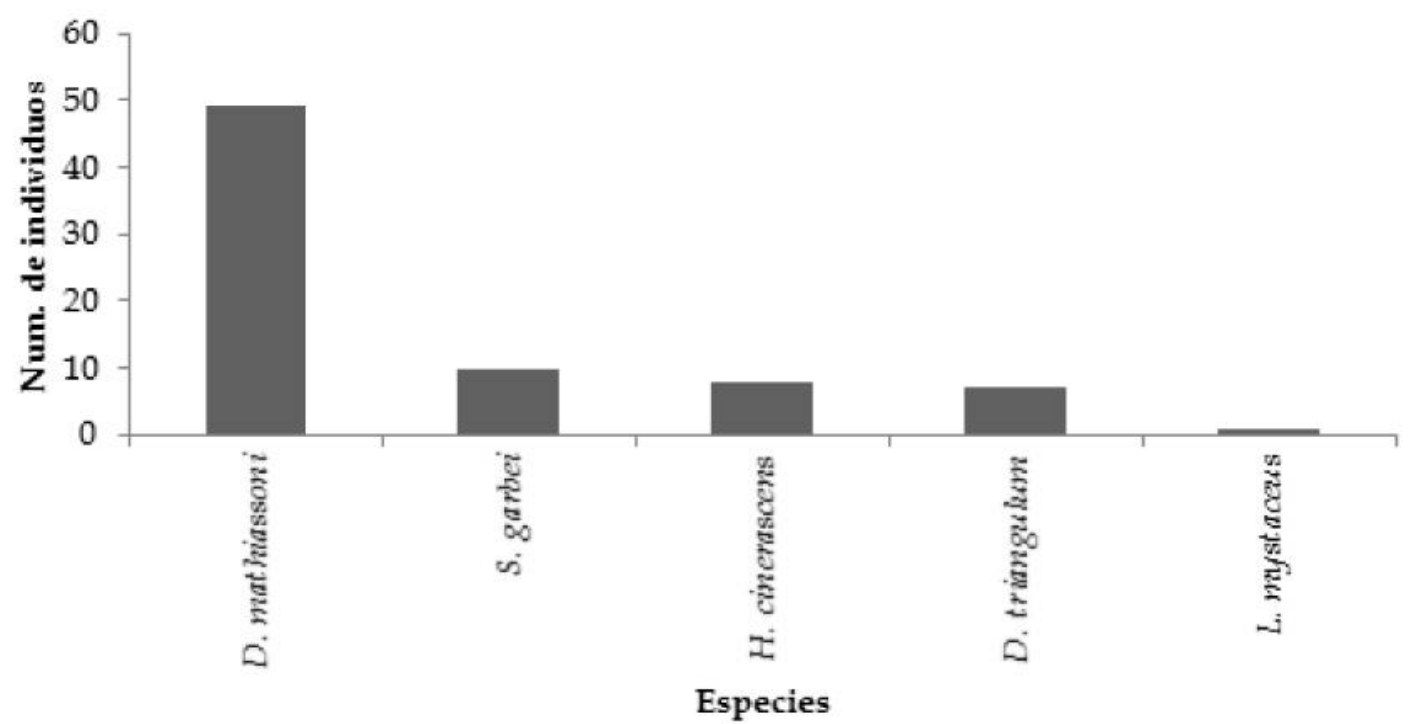

Figura 3. Distribución de la riqueza y abundancia de especies capturadas en el humedal de potrero del Centro de Investigaciones Amazónica Macagual, durante los meses de marzo y noviembre de 2012. 
tal de anuros presentes en este humedal, seguido por H. punctatus $(24,6 \%)$, D. mathiassoni $(18,8 \%)$, y las especies menos abundantes $H$. cinerascens $(10.1 \%)$ y Scinax garbey $(8,6 \%)$ (Figura 4$)$.

Sin embargo, la mayor dominancia, según el índice de Simpson (D), la obtuvo el HP. La mayor equitatividad (1-D) se encontró en el HBB. Mediante el índice de Margalef se obtuvo que la mayor riqueza la presentó el HBB, debido a la heterogeneidad vegetal, generando condiciones climáticas óptimas para los anuros, determinando nichos más restringidos y más especies por unidad de área. Estas condiciones favorecen la diversidad y abundancia de anuros porque les confieren diversos sitios de refugio, alimentación y oviposición (Lanno y Donnelly 2005) (Tabla 1).

\section{Discusión}

La riqueza de los humedales del estudio fue de cinco especies de anuros para cada uno, donde la presencia o ausencia de una especie en uno y otro humedal está sujeto al tipo de cobertura vegetal del área; evidencia de esto, están las especies $L$. mystaceus y $H$. punctatus que solo habitan como exclusivas en cada uno de los humedales. $L$. mystaceus solo fue encontrada en el HP, pues es una rana caracterizada porque habita zonas inundadas y abiertas, además de adaptarse a las modificaciones humanas de su hábitat (perturbaciones antrópicas) (Duellman y Trueb 1994). H. punctatus, propia del
Tabla 1. Comparación de los índices de abundancia, riqueza y diversidad de los anuros capturados en dos humedales (borde de bosque y potrero) del Centro de Investigaciones Macagual (CIMAZ), durante los meses de marzo y noviembre del 2012.

\begin{tabular}{lcc}
\hline Índice & \multicolumn{2}{c}{ Humedal } \\
\cline { 2 - 3 } & borde bosque & potrero \\
\hline Riqueza & 5 & 5 \\
Abundancia & 69 & 75 \\
Simpson D & 0,256 & 0,465 \\
Equitatividad 1 D & 0,744 & 0,535 \\
Shannon_H & 1,472 & 1,064 \\
Margalef & 0,945 & 0,927 \\
\hline
\end{tabular}

HBB, se halló aquí por ser individuos que habita los bosques primarios o secundarios pero por lo general salen a los pastos o arbustos cercanos (hojas y ramas) de las zonas inundadas, pantanos estacionales y superficies de agua en medio de áreas verdes (Duellman y Trueb 1994).

En cuanto a la complejidad de la estructura de los humedales, refiriéndonos a que cada uno presenta ciertas particularidades en cuanto a sus comunidades vegetales, brindando refugio a los diferentes animales que viven allí; el HBB ofrece mayor disponibilidad de alimento, refugio y sitios de reproducción, por lo cual es ocupado por distintas especies de anuros, explicando la mayor diversidad en este lugar; es decir, que aunque existe una misma riqueza (cinco especies) y una abundancia no muy signi-

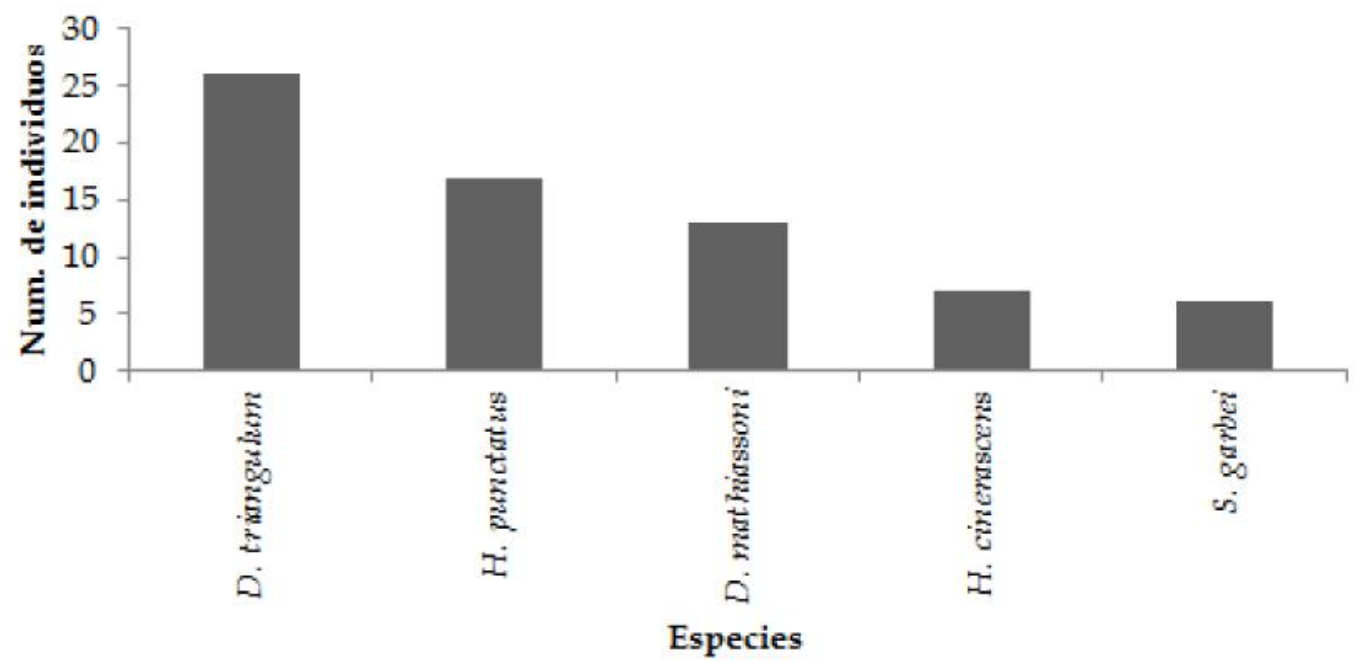

Figura 4. Distribución de la riqueza y abundancia de especies capturadas en el humedal de borde de bosque del Centro de Investigaciones Amazónica Macagual, durante los meses de marzo y noviembre de 2012. 
ficativa una de la otra en los dos humedales (HBB con 69 individuos y HP con 75), refleja el contraste de hábitats disponibles para la fauna de anuros y su grado de homogeneidad, la forma en la que menos individuos y con la misma riqueza se comparten mejor el hábitat y alimento (HBB).

La sustitución de los bosques naturales por potreros, áreas abiertas y libres de vegetación arbórea con poca vegetación herbácea, es hábitat preferido por D. mathiassoni (Rueda-Almonacid et al. 2004). La dominancia de esta especie para el HP, evidencia el grado de intervención al que está siendo sometido el humedal, debido a que esta rana habita lugares cercanos a los cuerpos de agua y zonas abiertas y en consecuencia es muy común en hábitats transformados, tolerante a los impactos humanos graves (Rueda-Almonacid 2010).

De igual forma es una especie de gran plasticidad y tolerancia ecológica, mostrando que sus requerimientos de hábitat no son exigentes, pues no solo se halló en el HP, sino también en el HBB, aunque es este último es de las especies con abundancias intermedias.

Por su parte, para el HBB, D. triamgulum fue la especie más representativa, la más abundante, pues estas ranas se hallan comúnmente alrededor de los humedales o estanques cerca al bosque para su refugio, y en la reproducción tienen lugar en estos cuerpos de agua, siempre ocupando la vegetación flotante donde forma coros (Duellman y Trueb 1994). Sin embargo, D. triamgulum se encontró también en el HP aunque en abundancia baja, quizás esto refleja que esta especie habita en una variedad de situaciones no forestales (Azevedo-Ramos et al. 2004). No obstante, sus abundancias se podrían ver afectadas poco a poco, debido a la desecación de los humedales.

El HP fue el que presentó una mayor abundancia y dominancia (0.465), con una mayor extensión del área y más fuente de agua, de manera que no solo funciona como refugio de anfibios, sino también de reptiles y mamíferos que necesitan de ambientes lóticos.

En cuanto al HBB, su abundancia fue menor (69 individuos), pero repartidas en la misma cantidad de especies que el otro humedal, lo que simbolizó que había una mejor distribución u homogeneidad del espacio y quizás alimento, presentó mayor diversidad y equitatividad. Este humedal es un ecosistema caracterizado principalmente por el refugio que los anuros tienen al lado del bosque y como zona para ovopositar en épocas de reproducción.

\section{Conclusiones}

Los anuros son un orden de gran importancia como indicadores del estado de intervención en los humedales; dentro del estudio se registró un total de 144 individuos, distribuidos en seis especies, cuatro géneros y dos familias (Hylidae y Leptodactylidae). El HP presentó la mayor abundancia, capturándose 75 individuos; la dominancia de D. mathiassoni, evidencia el grado de intervención al que está siendo sometido el humedal, sin embargo, por su mayor extensión de área y más fuente de agua, no solo funciona como refugio de anfibios, sino también de reptiles y mamíferos que necesitan de ambientes lóticos. En cuanto al HBB se capturaron 69 individuos pertenecientes a la familia Hylidae, la especie más representativa fue $D$. triamgulum, porque es una rana que se halla comúnmente alrededor de los humedales o estanques cerca al bosque para su refugio y su reproducción tienen lugar en estos cuerpos de agua, es un humedal que aporta una mejor distribución u homogeneidad del espacio y quizás alimento para las especies allí presentes. Por ello, la importancia de los humedales es el hecho de que sea hábitat de una gran cantidad de organismos en especies de flora y fauna; el refugio de muchos anfibios, demuestra el valor de estos ecosistemas para la región.

\section{Agradecimientos}

A la Universidad de la Amazonía, por la financiación del proyecto. Al Museo de Historia Natural de la Universidad de la Amazonia (UAM). Al Semillero de Investigación en Herpetología, por el préstamo del material requerido para los muestreos de anuros y a sus integrantes por el apoyo.

\section{Literatura citada}

Acosta-Galvis AR. 2000. Ranas, salamandras y caecilias (Tetrapoda: Amphibia) de Colombia. Biota Colomb. 1 (3): 289-319.

Alford-Ross A, Stephen-Richards J. 1999. Global amphibian declines: a problem n applied ecology. Ann Rev Ecol System 30 (1): 133-65. 
Azevedo-Ramos C, Coloma LA, Ron S, Acosta-Galvis AR. 2004. Dendropsophus triangulum. (En línea) 2013 (Acceso 23 de febrero) http://www.iucnredlist.org/details/ $55679 / 0$

Baber MJ, Babbitt KJ. 2003. The relative impacts of native and introduced predatory fish on a temporary wetland tadpole assemblage. Oecologia. 136: 289-95.

Beebee-Trevor J. 1996. Ecology and conservation of amphibians. Londres: Springer; 214 pp.

Begon M, Colin TR, Harper JL. 2005. Ecology: From individuals to ecosystems. Oxford: Blackwell; 1068 pp.

Castellanos CA. 2001. Los ecosistemas de humedales en Colombia. (Trabajo de grado). Especialista en Sistemas de Información Geográfica. Bucaramanga: Universidad Industrial de Santander.

Duellman EW, Trueb L. 1994. Biology of amphibians. London: McGraw Hill; 275 pp.

Fitzgerald-Lee A, Cruz FB, Perotti G. 1999. Phenology of a lizard assemblage in the Dry Chaco of Argentina. $J$ Herpetol. 33: 526-35.

Estrada-Gonzáles CA, Rosas-Patiño G. 2007. Proyecto: Centro de Investigaciones Amazónicas Macagual (CIMAZ). Caquetá: Universidad de la Amazonia.

Lanno MJ, Donnelly MA. 2005. Amphibian declines: The conservation status of United States Species. London: University of California; $915 \mathrm{pp}$.

Manzanilla J, Pefaur J. 2000. Consideraciones sobre métodos y técnicas de campo para el estudio de anfibios y reptiles. Rev Ecol Lat Am. 7: 19-30.
Moreno V, García JF, Villalba JC. 2007. Descripción general de los humedales de Bogotá. Bogotá: Sociedad Geográfica de Colombia y Academia de Ciencias Geográficas.

Marín-Henao D, Franco-Velásquez S. 2007. Informe de la caracterización de humedales en el proyecto conservación de humedales altoandinos en cinco municipios del departamento de Caldas: Neira, Salamina, Villamaría, Manizales y Marulanda. URL disponible en: http://www.academia.edu/7012741/Diagnostico _de_Los_Humedales_Altoandinos_y_de_Paramo_ de_Caldas

Rueda-Almonacid JV. 1999. Anfibios y reptiles amenazados de extinción en Colombia. Rev Acad Colomb Cien Exac Fis Nat. 14: 25-8.

Rueda-Almonacid JV, Lynch JD, Amezquita A. 2004. Libro rojo de los anfibios de Colombia. Bogotá: Conservación Internacional Colombiana, Universidad Nacional.

Rueda-Almonacid JV, Castro F, Acosta-Galvis A, Bolívar W. 2010. Dendropsophus mathiassoni. The IUCN Red List of Threatened Species. Version 2014.1. [20 November 2013]. URL disponible en: http:www.iucnredlist.org

Scott NJ. 1994. Complete species inventories. En: Heyer WR, Donnelly MA, McDiarmid RW, Hayek LC, Foster MS (eds.). Measuring and monitoring biodiversity: Standard methods for amphibians. Washington, DC: Smithsonian Institution Press; pp. 78-84.

Urbina-Cardona JN, Cáceres-Andrade SP. 2009. Ensamble de anuros de sistemas productivos y bosques del piedemonte Llanero, departamento del Meta Colombia. Caldasia. 31 (1): $175-94$. 